\title{
Efecto del flunixin meglumine en el porcentaje de gestación de ovejas receptoras de embriones.
}

\section{Artículo Original}

Jesús R. Aké-López ${ }^{1}$, Jorge A. Quintal-Franco², José C. Segura-Correa1.

${ }^{1}$ Departamento de Reproducción y Mejoramiento Genético, Facultad de Medicina Veterinaria y Zootecnia, Universidad Autónima de Yucatán. ${ }^{2}$ Departamento de Reproducción Animal, Campo Experimental Mocochá, INIFAP, Yucatán, México.

\section{RESUMEN.}

Introducción. El porcentaje de gestación después de la transferencia embrionaria puede ser afectado por la manipulación que se realiza con los embriones, lo cual retrasa su desarrollo y afecta el adecuado reconocimiento de la gestación. Una alternativa para mejorar la tasa de gestación es alargar la vida del cuerpo lúteo a través de fármacos que impidan la secreción de la prostaglandina F2a. Objetivo. Evaluar el efecto del Flunixin Meglumine (FM) en el porcentaje de gestación, duración de la fase lútea y prolificidad de ovejas receptoras de embriones.

Material y Métodos. Las ovejas receptoras recibieron dos embriones y se asignaron al azar a uno de dos grupos: FM-2: Ovejas $(\mathrm{n}=10)$ tratadas con $2.2 \mathrm{mg} / \mathrm{Kg}$ de peso vivo de FM dos veces al día (06:00 y 18:00 hrs); testigo: ovejas $(n=10)$ tratadas con $1.5 \mathrm{ml}$ de agua estéril una vez al día (06:00 hrs). Ambos grupos se trataron durante ocho días, a partir del quinto día postransferencia. Del día 15 al 19 se tomaron muestras de sangre para determinar la concentración de progesterona. Resultados. El porcentaje de gestación del grupo testigo fue $50 \%$ y de las ovejas del grupo FM-2 $40 \%$ ( $>>0.05)$. La prolificidad fue similar $(\mathrm{p}>0.05)$ entre el grupo testigo y el grupo FM-2 (1.4 vs 1.25 corderos). La fase lútea fue más larga para el grupo FM-2 que para el grupo testigo (18.1 vs 16.2 días; $\mathrm{p}<0.05)$

Conclusión. La aplicación del FM dos veces al día, no modificó la tasa de gestación y prolificidad de las ovejas receptoras, a pesar de que retrasó la luteolisis. (Rev Biomed 2002; 13:100-108)

Palabras clave: Gestación, embriones, ovejas Pelibuey, Flunixin Meglumine.

Solicitud de sobretiros: Dr. Jesús R. Aké-López. Departamento de Reproducción Animal, Facultad de Medicina Veterinaria y Zootecnia, Universidad Autónoma de Yucatán. Km 15.5 carretera Mérida-Xmatkuil, A.P. 4-116 Itzimná, Mérida, Yucatán, México.

Teléfono: (999) 94232 13; Fax: (999) 94232 05; Email: alopez@tunku.uady.mx

Recibido el 14/Febrero/2001. Aceptado para publicación el 21/Junio/2001. 


\section{JR Aké-López, JA Quintal-Franco, JC Segura-Correa.}

\section{SUMMARY.}

\section{Effect of Flunixin Meglumine on gestation rate of embryo recipient ewes.}

Introduction. Gestation rate after embryo transfer can be affected by handling performed during transferring procedures, which negatively influences embryo development. Such a situation interferes with an adequate maternal recognition of pregnancy which results in luteolysis and embryo losses. One alternative to improve gestation rate in embryo recipient females is by lengthening corpus luteum lifespan. The latter can be achieved through treatment with drugs that inhibit synthesis and secretion of prostaglandin $\mathrm{F}_{2}$ to block luteolysis.

Objective. The aim of this study was to evaluate the effect of Flunixin Meglumine(FM) on the duration of luteal phase, gestation rate, and prolificacy of embryo receptor ewes.

Materials and Methods. Twenty ewes were used as recipients and upon embryo transfer were assigned to one of two treatments: FM2 ewes (n $=10)$ were treated twice daily (06:00 and 18:00 h) with $2.2 \mathrm{mg} / \mathrm{kg}$ of Flunixin Meglumine (Fynadine, Schering Plough); Controls ( $n=10$ ewes) which received a vehicle injection once daily $(06: 00 \mathrm{~h})$. Both groups were treated for 8 days, starting on day 5 after embryo transfer. Blood samples were taken daily in order to quantify circulating concentrations of progesterone from day 15 to 19 after estrus.

Results. Gestation rate of Control group was 50\% and FM2 was $40 \%(\mathrm{p}>0.05)$. Prolificacy was similar ( $p>0.05)$ between Controls and FM2 ((1.4 vs. 1.25 lambs/ewe respectively). Length of luteal phase was greater in ewes of the FM2 group than in the Controls (18.1 vs. 16.2 days respectively; $\mathrm{p}<0.05$ )

Conclusion. Treatment with Flunixin Meglumine $(2.2 \mathrm{mg} / \mathrm{kg}$ ) twice daily had no effect on gestation rate and prolificacy of embryo recipient ewes, even though it delayed luteolysis.

(Rev Biomed 2002; 13:100-108)
Key words: Gestation, embryo, Pelibuey ewes, Flunixin Meglumine.

\section{INTRODUCCIÓN.}

La transferencia de embriones (TE) en el ganado es una herramienta de gran utilidad que permite utilizar al máximo la capacidad reproductiva de las hembras de gran valor genético. A pesar de ello, la TE tiene diversos obstáculos que no permiten obtener buenos resultados de su uso, entre ellos se puede mencionar la baja tasa de sobrevivencia de los embriones a la transferencia (1-3).

Uno de los principales factores que interfieren con la sobrevivencia de los embriones transferidos, y que también se presenta bajo condiciones de apareamiento natural, es la adecuada sincronía materno-embrionaria (4-6)

En las ovejas el mantenimiento de la gestación depende de que la secreción de progesterona por el cuerpo lúteo se mantenga, esto como resultado de la prevención de la luteólisis. Para evitar la lisis del cuerpo lúteo y por consiguiente la disminución de los niveles de progesterona, el embrión debe indicar al útero su presencia a través de la secreción de la proteína trofoblástica ovina (oTP-1). A este evento se le conoce como "reconocimiento materno de la gestación" $(5,7,8)$.

Una de las causas más comunes de pérdidas embrionarias se presenta como consecuencia de una falla en el reconocimiento materno de la gestación, la cual puede deberse a un retraso en el desarrollo embrionario, que resulta en una asincronía en la emisión-recepción de la proteína trofoblástica, o a un adelanto en la liberación de la Prostaglandina F2 (PGF2) por parte de la madre $(7,9,10)$. En ambas situaciones, se presenta la síntesis y liberación de la PGF2, lo que provoca la lisis del cuerpo lúteo y por tanto la pérdida del embrión. Entre los factores más importantes que retrasan el desarrollo embrionario se encuentran el estrés térmico y la manipulación del embrión, esto último necesariamente se realiza durante la transferencia

\section{Revista Biomédica}




\section{Efecto del flunixin meglumine en gestación de ovejas receptoras de embriones.}

de los mismos a la hembras receptoras $(9,11,12)$

Se han planteado diversas estrategias para alargar la vida del cuerpo lúteo, una de estas alternativas es el uso del Flunixin Meglumine (FM), la cual es una potente droga antiflamatoria no esteroide que inhibe la enzima ciclo-oxigenasa $(13,14)$. La mayoría de los estudios demuestran que su aplicación evita eficazmente la formación de la PGF2a por el útero $(14,15)$. Aunque su efecto antiprostaglandínico (y antiluteolítico) ha sido demostrado, el uso del FM para prevenir la destrucción del cuerpo lúteo alrededor del momento en que se produce el reconocimiento de la gestación es limitado, por lo que es necesario realizar más estudios al respecto.

El objetivo del presente estudio fue investigar si la aplicación del Flunixin Meglumine en la fase lútea media y tardía, retrasa la luteolisis y si permite incrementar el porcentaje de gestación y prolificidad de las receptoras de embriones.

\section{MATERIAL Y MÉTODOS.}

Localización. El presente trabajo se realizó en las instalaciones del Campo Experimental Mocochá dependiente del INIFAP-SAGARPA y en el Departamento de Reproducción Animal de la Facultad de Medicina Veterinaria y Zootecnia (FMVZ) de la Universidad Autónoma de Yucatán, en el periodo comprendido de febrero de 1998 a junio de 1999. El Campo Experimental (21 06' de latitud Norte y 89 27' de longitud Oeste) y la FMVZ (20 57' de latitud Norte y 89 39' de longitud Oeste) están situados en la zona centro del estado de Yucatán, México. El clima de la región se clasifica como tropical subhúmedo, correspondiente al tipo Aw0 (16). Se encuentra a 7-8 metros sobre el nivel del mar, la temperatura promedio anual oscila entre 25 y $28^{\circ} \mathrm{C}$; la humedad relativa media anual es de alrededor de $75-80 \%$ y la precipitación pluvial anual promedio es de $900 \mathrm{~mm}$, distribuyéndose principalmente entre los meses de junio a octubre (17).

Animales. Se utilizaron 30 ovejas de raza
Pelibuey (10 donadoras y 20 receptoras) las cuales se seleccionaron de acuerdo a las siguientes características:

a) Condición corporal: entre 3 y 4 puntos, en una escala 1-5 (18).

b) Amamantamiento: que ninguna de las ovejas estuviera amamantando a sus crías.

c) Tiempo posparto: tener cuando menos 90 días posparto.

d) Número de parto: se eligieron ovejas entre 2 y 4 partos.

Los animales se mantuvieron en pastoreo en zacate estrella (Cynodon nlemfuensis) por espacio de 6-7 horas, se les suministró 250 gr/oveja/día de un suplemento comercial con $14 \%$ de proteína cruda y recibieron agua y sales minerales $a d$ libitum.

Superovulación, colección y transferencia de embriones. El tratamiento superovulatorio de las ovejas donadoras $(n=10)$ se inició el día 9-10 del ciclo estral (estro=día 0), utilizando 500 UI de hormona folículo estimulante y hormona luteinizante porcina (Pluset; Serono, México) por oveja, que se aplicó dos veces por día (a intervalos de $12 \mathrm{~h}$ ) en dosis decrecientes durante cuatro días (100-100, 75-75; 50-50 y 25-25 UI). Con la finalidad de inducir y sincronizar la presentación del estro, al tercer día de iniciado el tratamiento superovulatorio, a las donadoras se les aplicó dos inyecciones de $7.5 \mathrm{mg}$ de $\mathrm{PGF}_{2}$ a (Prosolvin; Intervet, México) por oveja, con un intervalo de 12 horas. Con la finalidad de sincronizar el estro receptora-donadora, el mismo día que se aplicó la prostaglandina a las donadoras, se retiró el implante subcutáneo impregnado con norgestomet (Crestar; INTERVET-México) a las receptoras, los cuales se colocaron con 9 días de anterioridad; al momento del retiro del implante se inyectó intramuscularmente 150 UI de Gonadotropina Coriónica Equina (Folligon, INTERVET-México) por oveja. Las donadoras recibieron monta natural a las 0,12 y 24 horas de iniciado el estro, utilizando para ello sementales evaluados previamente en 


\section{JR Aké-López, JA Quintal-Franco, JC Segura-Correa.}

cuanto a su calidad seminal.

La recolección de los embriones se llevó a cabo por el método quirúrgico, a través de una laparotomía media ventral (19) el día seis después del primer servicio. Se utilizó como medio de lavado solución amortiguada con fosfatos (Dulbecco's PBS; Gibco-EUA) suplementada con $1 \%$ de suero fetal bovino. Una vez terminada la recolección de los embriones, el útero fue colocado nuevamente en la cavidad abdominal y se aplicó antibióticos (Engemicin 10\%, Intervet, México) y $7.5 \mathrm{mg}$ de Prosolvin.

La búsqueda de los embriones se realizó en el medio de recolección el cual se vertió en cajas Petri. Los embriones fueron evaluados con base en su estado de desarrollo y calidad morfológica utilizando los criterios descritos por Lindner y Wright (20).

Para la transferencia se seleccionaron los embriones en estadio de mórula y blastocisto de excelente y buena calidad, los embriones fueron transferidos en el último tercio del cuerno uterino ipsilateral al cuerpo lúteo de cada oveja receptora (19).

Tratamientos experimentales. Las ovejas receptoras $(n=20)$ recibieron por transferencia quirúrgica, el mismo día de la recolección (día 6), dos embriones del mismo estadio, y se asignaron al azar a uno de dos tratamientos, quedando de la siguiente forma:

Grupo FM-2, el cual se tratado con Flunixin Meglumine (FINADYNE; Schering Plough, México). Las ovejas $(n=10)$ de este grupo recibieron dos inyecciones diarias, en forma intramuscular, de $2.2 \mathrm{mg}$ de Flunixin Meglumine por $\mathrm{Kg}$ de peso vivo, una por la mañana (06:00 horas) y otra por la tarde (18:00 horas) durante 8 días, comenzando a partir del quinto día después de la transferencia de los embriones (día 11 después del estro).

Grupo Testigo, a estas ovejas $(\mathrm{n}=10)$ se les inyectó intramuscularmente $1.5 \mathrm{ml}$ de agua estéril una vez al día por la mañana (06:00 horas) durante 8 días, comenzando a partir del quinto día después de la transferencia de los embriones (día 11 después del estro).

Con la finalidad de detectar a los animales que no quedaron gestantes, cinco días después de la transferencia de los embriones se detectó el estro dos veces al día (06:00-07:00 y 17:00-18:00 horas), contando con la ayuda de machos enteros provistos de mandil, este manejo se realizó durante 15 días.

Muestreo Sanguíneo. Para cuantificar la concentración de progesterona plasmática de las ovejas receptoras, a todas ellas se les tomaron muestras de sangre del $15^{\circ}$ al $19^{\circ}$ día después del estro. Este esquema de muestreo fue diseñado para evaluar si el cuerpo lúteo observado el día de la transferencia de los embriones (día 6 del ciclo) permaneció funcional, más allá de la duración normal de un ciclo estral típico para la especie.

La sangre se colectó vía punción de la vena yugular en tubos vacutainer con anticoagulante (EDTA 10\%) y se centrifugó a 3000 rpm/15 minutos para separar el plasma, el cual una vez obtenido se congeló a $-20{ }^{\circ} \mathrm{C}$. La concentración de progesterona se determinó mediante radioinmunoensayo de fase sólida (Coat-A-Count, Diagnostic Products Corporation. Los Angeles, Ca. EUA.). El método fue altamente específico para la progesterona, con $0.2 \%$ de reacción cruzada con 20 Dihidroxiprogesterona y 3.4\% con 17 Hidroxiprogesterona; el límite de detección fue $0.03 \mathrm{ng} / \mathrm{ml}$. El coeficiente de variación intraensayo e interensayo fue de $6 \%$ y $9.5 \%$, respectivamente.

Análisis estadístico. Las variables de respuesta consideradas en este estudio fueron: 1.- Animales gestantes o no gestantes en los diferentes grupos. 2.- Prolificidad (número de corderos por hembra parida) de los diferentes tratamientos. La frecuencia de ovejas gestantes por grupo se analizó mediante una prueba exacta de Fisher. Para evaluar las diferencias en la prolificidad y la duración de la fase lútea entre los diferentes grupos, se utilizó un diseño completamente al azar, considerando como fuente de variación el efecto del tratamiento. La comparación entre las medias se realizó utilizando la prueba de Tukey. Todos los análisis estadísticos

\section{Revista Biomédica}


Efecto del flunixin meglumine en gestación de ovejas receptoras de embriones.

se hicieron utilizando el paquete estadístico SAS (21).

\section{RESULTADOS.}

El porcentaje de gestación de las ovejas del grupo testigo fue ligeramente superior al de las ovejas tratadas con Flunixin Meglumine (50 vs 40 $\%$ respectivamente); sin embargo, no se encontró diferencia estadística $(\mathrm{p}>0.05)$ entre los dos tratamientos (cuadro 1).

En el cuadro 2, se presenta la prolificidad de las ovejas tratadas con Flunixin Meglumine dos veces al día y del grupo testigo, no se encontró diferencia significativa ( $p>0.05)$ entre los grupos.

\section{Cuadro 1}

Porcentaje de gestación de las ovejas Pelibuey tratadas con Flunixin Meglumine y testigo, a las cuales se les transfirieron dos embriones.

\begin{tabular}{|c|c|c|c|}
\hline Tratamiento & $\mathrm{N}$ & Ovejas Gestantes & Porcentaje \\
\hline Testigo & 10 & 5 & $50.0 \mathrm{a}$ \\
\hline $\begin{array}{l}\text { Flunixin dos } \\
\text { veces al día }\end{array}$ & 10 & 4 & $40.0 \mathrm{a}$ \\
\hline
\end{tabular}

a.- No se encontró diferencia significativa entre tratamientos $(\mathrm{p}>0.05)$

\section{Cuadro 2}

Frecuencia por tipo de parto y prolificidad de ovejas Pelibuey receptoras de embriones tratadas con Flunixin Meglumine y del grupo testigo.

\begin{tabular}{|c|c|c|}
\hline Tratamiento & $\mathrm{n}$ & $\begin{array}{l}\text { Partos Partos Prolificidad } \\
\text { Simples Dobles }\end{array}$ \\
\hline
\end{tabular}

\begin{tabular}{lllll}
\hline Testigo & 5 & 3 & 2 & $1.4^{\mathrm{a}}$ \\
Flunixin dos veces al día & 4 & 3 & 1 & $1.25^{\mathrm{a}}$ \\
\hline
\end{tabular}

a.- No se encontró diferencia significativa entre tratamientos $(\mathrm{p}>0.05)$

La concentración de progesterona plasmática (entre los días 15 y 19) individual de las ovejas testigo y las tratadas con Flunixin Meglumine, que no gestaron, se presenta en el cuadro 3. En este cuadro se puede observar que en dos de las ovejas tratadas con Flunixin la concentración de progesterona se mantuvo por arriba de $1 \mathrm{ng} / \mathrm{mL}$ hasta el día 19 que fue el último día de muestreo; en las otras cuatro el descenso ocurrió a los 17.7 días en promedio, el promedio global en este grupo fue de 18.1 días. En las ovejas del grupo testigo el descenso de la progesterona se dió a los 16.2 días en promedio, la diferencia encontrada entre los dos grupos fue estadísticamente significativa $(\mathrm{p}<0.05)$.

\section{DISCUSIÓN.}

Los resultados de este trabajo muestran que el tratamiento con Flunixin Meglumine aplicado dos veces al día provocó, en las ovejas que no gestaron, un alargamiento de 1.9 días en la fase lútea en comparación con las ovejas testigo. Este resultado es similar a lo encontrado en experimentos previos $(22,23)$ en donde la fase lútea se alargó más allá de la duración normal para la especie ovina, lo cual refuerza el hecho de que el Flunixin es eficaz para retrasar la luteólisis, tal y como se reporta en otros estudios $(15,24,25)$.

En el presente estudio a pesar de que la aplicación del Flunixin fue eficaz para alargar la fase lútea, el porcentaje de gestación de las ovejas tratadas no fue diferente $(\mathrm{p}>0.05)$ al porcentaje encontrado en el grupo testigo (50\% vs $40 \%$ ). Este resultado, de cierta forma indica que el alargar la fase lútea no es suficiente para incrementar el porcentaje de gestación de las ovejas receptoras, situación que se ha sugerido en otros estudios (26, 27).

Los resultados aquí encontrados son similares a los reportados por Odensvick y Gustafsson (26) los cuales trabajando con vacas, encontraron que la aplicación del Flunixin Meglumine fue eficaz para evitar la luteólisis y alargar la fase lútea de las vacas receptoras de embriones; sin embargo, no influyó sobre el porcentaje de gestación de los embriones transferidos con 3 días de asincronía. La diferencia entre el estudio de Odensvick y Gustafsson (26) y el presente, aunque en principio se realizó en 
JR Aké-López, JA Quintal-Franco, JC Segura-Correa.

Cuadro 3

Concentraciones plasmáticas $(\mathrm{ng} / \mathrm{ml})$ de progesterona $\left(P_{4}\right)$ de las ovejas Pelibuey receptoras que no gestaron después de la transferencia de embriones, tratadas con Flunixin Meglumine (FM) y del grupo testigo (T).

\begin{tabular}{llccccc}
\hline Oveja & Trat & $\begin{array}{c}\mathrm{P}_{4} \\
\text { Día } 15\end{array}$ & $\begin{array}{c}\mathrm{P}_{4} \\
\text { Día 16 }\end{array}$ & $\begin{array}{c}\mathrm{P}_{4} \\
\text { Día 17 }\end{array}$ & $\begin{array}{c}\mathrm{P}_{4} \\
\text { Día 18 }\end{array}$ & $\begin{array}{c}\mathrm{P}_{4} \\
\text { Día 19 }\end{array}$ \\
\hline 207 & $\mathrm{~T}$ & 0.10 & 0.10 & 0.10 & 0.10 & 0.10 \\
$2-2$ & $\mathrm{~T}$ & 3.6 & 3.0 & 1.4 & 0.4 & 0.12 \\
$4-2$ & $\mathrm{~T}$ & 4.6 & 2.0 & 0.48 & 0.12 & 0.10 \\
$5-14$ & $\mathrm{~T}$ & 2.8 & 0.6 & 0.2 & 0.18 & 0.10 \\
$5-5$ & $\mathrm{~T}$ & 0.10 & 0.10 & 0.10 & 0.9 & 3.2 \\
& & & & & & \\
$2-13$ & $\mathrm{FM}$ & 3.35 & 3.65 & 2.85 & 4.6 & 2.9 \\
217 & $\mathrm{FM}$ & 3.3 & 1.3 & 1.2 & 0.42 & 0.15 \\
227 & $\mathrm{FM}$ & 2.9 & 3.2 & 1.4 & 0.9 & 0.1 \\
$2-5$ & $\mathrm{FM}$ & 5.0 & 3.7 & 0.15 & 0.11 & 0.18 \\
$3-3$ & $\mathrm{FM}$ & 2.0 & 2.3 & 1.1 & 1.6 & 1.2 \\
& $\mathrm{FM}$ & 3.3 & 2.6 & 1.2 & 0.6 & 0.1 \\
\hline
\end{tabular}

especies diferentes, radica en el tiempo empleado para la transferencia de los embriones. En este estudio los embriones que se transfirieron tenían como máximo 18 horas de asincronía, mientras que en el caso del trabajo de Odensvick y Gustafsson (26) los embriones tenían 3 días de asincronía.

Odensvick y Gustafsson (26) concluyen que los eventos que están involucrados en el reconocimiento materno de la gestación son muy complejos y que no son fácilmente influenciados por substancias exógenas; sin embargo, no excluyen que el uso del Flunixin Meglumine pueda ser benéfico en la transferencia de embriones, proporcionando soporte al cuerpo lúteo durante los períodos críticos, que para el caso de los ovinos es alrededor del los días 12 y13 del ciclo, que es cuando se debe realizar el reconocimiento materno de la gestación $(7,28)$

Mejía y col. (27) trabajando con ovejas, transfirieron dos embriones por oveja receptora las cuales tenían, en promedio, una asincronía de 3.5 días, en relación a las donadoras y obtuvieron resultados similares a los encontrados en este estudio. En los resultados reportados por Mejía y col. (27) se observa que la aplicación de los tratamientos empleados fueron eficaces para retrasar la luteólisis y alargar la fase lútea (además Revista Biomédica de incrementar la concentración de progesterona); sin embargo, no tuvieron ningún efecto significativo sobre el porcentaje de gestación.

En cuanto a la falta de diferencia entre el grupo tratado con Flunixin y el grupo testigo de este estudio, es probable que el tratamiento con Flunixin no fue lo suficientemente eficaz como para incrementar el porcentaje de gestación, además hay que considerar que el tamaño de la muestra empleado en este estudio probablemente no permitió evaluar adecuadamente esta diferencia. Por otro lado, los bajos porcentajes de gestación encontrados en las ovejas de este trabajo probablemente se deba, como se reporta para otros estudios $(12,29)$ a una asincronía entre el embrión y el medio ambiente uterino; en parte esta asincronía puede estar dada por retraso en el desarrollo del embrión como consecuencia de la manipulación en el proceso de recolección, evaluación y transferencia a las receptoras.

Hay que considerar que en este estudio, no se controló en forma estricta el tiempo de asincronía de los embriones transferidos, aunque ésta no fue mayor a 18 horas. Este es un aspecto que hay que considerar, ya que se reporta que bajo condiciones de trópico, con ovejas Pelibuey, la sincronía puede ser un factor de mucha importancia $(30,31)$. 


\section{Efecto del flunixin meglumine en gestación de ovejas receptoras de embriones.}

Novelo (31) encontró que ovejas receptoras que reciben embriones con una sincronía entre el estro de la donadora y de la receptora de \pm 12 horas (hasta 12 horas antes y hasta 12 horas después) presentan mayor porcentaje de gestación (58.3\%; $\mathrm{p}<0.05)$ que las ovejas que reciben embriones (20\%) con una asincronía mayor a 12 horas (una diferencia mayor a 12 horas antes o después del inicio del estro de las donadoras). Por otra parte, también es conveniente mencionar que el número de animales empleados en este estudio no fue lo suficientemente grande para encontrar diferencias entre el porcentaje de gestación.

Otro factor que pudo influir en los resultados de este estudio es la temperatura del medio ambiente. El experimento en cuestión se realizó en el mes de marzo y abril, meses que se caracterizan por una elevada temperatura ambiental. Diversos trabajos han sugerido que temperaturas altas puede afectar la sobrevivencia embrionaria a través de un efecto directo sobre el embrión o bien por un efecto indirecto $(2,9,32$, 33). Aunque no se puede asegurar, cabe la posibilidad de que la temperatura ambiental alta afectó negativamente al embrión, el cual muere como consecuencia del daño que recibió con anterioridad. Por último, también existe la posibilidad que los bajos porcentajes de gestación obtenidos en ambos grupos sean debidos al manejo que se realizó para dar el tratamiento a las ovejas así como en el proceso de superovulación, recolección y manipulación de los embriones para su transferencia.

En cuanto a la prolificidad obtenida en el grupo tratado y el grupo testigo, no se observó diferencia y el promedio obtenido se encuentra dentro de los promedios para la raza Pelibuey (34), este resultado era de esperar ya que este promedio es poco modificable por el tratamiento con Flunixin Meglumine, el cual ejerce su efecto sobre la vida del cuerpo lúteo $(14,15,24)$ y no sobre la tasa de ovulación.

En conclusión, en el presente estudio se observó que el tratamiento con Flunixin Meglumine dos veces al día, fue efectivo para retrasar la luteólisis; sin embargo no mostró ningún efecto sobre el porcentaje de gestación y prolificidad de las ovejas receptoras de embriones.

\section{AGRADECIMIENTOS.}

Se agradece al Consejo Nacional de Ciencia y Tecnología (CONACYT), por el apoyo brindado (Registro 12100) para la elaboración de este trabajo. También se agradece al Laboratorio Schering Plough por la donación del Fynadine utilizado.

\section{REFERENCIAS.}

1.- Putney JD, Thatcher WW, Drost M, Wrigh JM, DeLorenzo MA. Influence of environmental temperature on reproductive performance of bovine embryo donors and recipients in the southwest region of the United States. Theriogenology 1986; 30:905-22.

2.- Sreenan MJ, Diskin GM. Early embryonic mortality in the cow: its relationship with progesterone concentration. Vet Rec 1983; 28:517-20.

3.- Mutiga RE. Increasing reproductive rates in tropical sheep by means of embryo transfer. Theriogenology 1991; 36:681-8.

4.- Thatcher WW, Bazer FW, Sharp DC, Roberts RM. Interrelations between uterus and conceptus to maintain corpus luteum function during early pregnancy, Sheep, Cattle, pig and horses. J Anim Sci 1986; 67 (suppl. 2):4761.

5.- Garverick HA, Zollers WG, Smith MF. Mechanisms associated with corpus luteum lifespan in animals having normal or subnormal luteal function. Anim Reprod Sci $1992 ; 28: 111-24$.

6.- Thatcher WW, Staples CR, Danet-Desnoyers G, Oldick B, Schmitt EP. Embryo Health and mortality in sheep and cattle. J Anim Sci 1994; 72 (suppl.): 16-30.

7.- Roberts RM, Schalue-Francis T. Maternal recognition of pregnancy and embryonic loss. Theriogenology 1990; $33: 175-83$.

8.- Al-guboruy HK, Abdennebi L. Evidence that the conceptus contributes to the inhibition of follicular grow in the ewe. Anim Reprod Sci 1996; 45:71-80. 


\section{JR Aké-López, JA Quintal-Franco, JC Segura-Correa.}

9.- Putney JD, Drost M, Thatcher WW. Embryonic development in superovulated dairy cattle exposed to elevated between days 1 to 7 post insemination. Theriogenology 1988; 30:195-209.

10.- Hunter MG, Ayad VJ, Gilbert CL, Shouthee JA, Wathes DC. Role of prostaglandin F2 alpha and oxytocin in the regression of GnRH-induced abnormal corpora lutea in anoestrous ewes. J Reprod Fertil 1989; 85:551-61.

11.- Wilmut I, Sales DI, Ashworth CJ. Maternal and embryonic factors associated with prenatal loss in mammals. J Reprod Fertil 1980; 76:851-64

12.- Pope WF. Uterine asynchrony: A cause of embryonic loss. Biol Reprod 1988; 39:999-1003

13.- Hardee GE, Smith JA, Harris SJ. Pharmacokinetics of Flunixin Meglumine in the cow. Res Vet Sci 1985; 39:110-12.

14.- Odensvick K, Cort N, Basu S. Effect of Flunixin Meglumine on prostaglandin F2alpha synthesis and metabolism. J Vet Pharmacol Therapeut 1989; 12:307-11.

15.- Aiumlamai S, Fredriksson G, Kindahl H, Uggla A, Edqvisy LE. The effect of Toxoplasma gondii infection in Flunixin Meglumine treated pregnant ewes as monitored by plasma levels of 15-Ketodihidroprostaglandin F2 alpha, progesterone, oestrone sulphate and ultrasound scanning. J Vet Med 1990; 37:23-34.

16.- García E. Modificaciones al sistema de Clasificación Climática de Koppen. Instituto de Geografía. México: Universidad Autónoma de México; 1973. p. 246.

17.- INEGI. Monografía del estado de Yucatán. Mérida: Instituto Nacional de Estadística, Geografía e Informática y Gobierno del Estado de Yucatán, Secretaria de Planeación; 1993.

18.- Russel A. Body condition scoring of sheep. In practice 1984; May:91-93

19.- Ake LR, Alfaro GM, Herrera CJ. Técnica de ovulación múltiple y transferencia de embriones. En: Manual del curso de teórico-práctico de ovulación múltiple y trasplante de embriones en ovinos. Mocochá: UADY-FMVZ, INIFAPC.E.; 1988. p. 107-32.

20.- Lindner MG, Wright WR. Bovine embryo morphology and evaluation. Theriogenology 1983; 20:407-16.
21.- SAS Institute Inc. SAS/STAT User's Guide. Release 6.03 Edition. SAS Institute 1990. Cary, NC, USA.

22.- Aké LR, Segura CJ., Quintal FJ, Heredia AM. Duración del ciclo estral y fase lútea de ovejas Pelibuey tratadas con Flunixin Meglumine". Memorias de la XXXV Reunión Nacional de Investigación Pecuaria Yucatán 99. Mérida, Yucatán, Octubre 29-22, 1999. p. 19

23.- Aké LR, Segura CJ, Quintal FJ. Efecto del Flunixin Meglumine en la fertilidad, duración del ciclo estral y fase lútea de ovejas Pelibuey bajo condiciones de trópico. Memorias de la XXXV Reunión Nacional de Investigación Pecuaria Yucatán 99. Mérida, Yucatán, Octubre 29-22, 1999. p. 20.

24.- Odensvick K, Aiumlamai S, Kindahl H. Effect of Flunixin Meglumine on prostaglandin F2 metabolite levels in the heifer. Acta Vet Scand 1991; 87:264-66.

25.- Odensvick K. Pharmakicinetics of Flunixin and its effect on prostaglandin F2alpha metabolite concentrations after oral and intravenous administration in heifers. J Vet Pharmacol Therapeut 1995; 18:254-59.

26.- Odensvick K, Gustafsson H. Effect of Flunixin during asynchronous embryo transfer in the heifer. Anim Reprod Sci 1994; 36:13-24.

27.- Mejia VO, Zarco QL, Rojas MS, Rosas GM, Valencia MJ. Efecto del líquido folicular equino y la somatotropina bovina en la sobrevivencia de embriones ovinos transferidos asincrónicamente. Vet Méx 1998; 29:359-67.

28.- Godkin JD, Bazer FW, Thatcher WW, Roberts RM. Proteins released by cultured day 15-16 conceptuses prolong luteal maintenance when introduced into the uterine lumen of cyclic ewes. J Reprod Fertil 1984; 71:57-64.

29.- Lawson RAS, Parr RA, Cahill LP. Evidence for maternal control of blastocyst growth after asynchronous transfer of embryos to the uterus of the ewe. J Reprod Fertil 1983; 67:477-83.

30.- Aké-López JR, Alfaro-Gamboa ME, Medina-Zaldivar M, Holy L. Porcentajes de preñez en hembras bovinas receptoras de embriones en el trópico. Agrociencia 1998; 32:165-8.

31.- Novelo-Ayuso EA. Efecto de dos tipos de hormonas sobre la respuesta superovulatoria, y de la sincronía del estro entre la receptoras y las donadoras sobre el porcentaje de gestación de ovejas Pelibuey bajo condiciones de trópico.

\section{Revista Biomédica}




\section{Efecto del flunixin meglumine en gestación de ovejas receptoras de embriones.}

Tesis de licenciatura. Mérida, Yuc. México: Universidad Autónoma de Yucatán FMVZ; 1999.

32.- Collier JR; Doelger GS; Head HH; Thatcher WW, Wilcox JC. Effects of heat stress during pregnancy on maternal hormone concentrations, calf birth weight and postpartum milk yield of Holstein cows. J Anim Sci 1982; 54:309-19.

33.- Drost M, Thatcher WW. Reducing embryo death in cattle. Proceedings of bovine short curse. American College of Theriogenologist and Society for Theriogenology; 1995. p. 133-140.

34.- Rodríguez RO. Manejo reproductivo. En Castellanos Ruelas AF. y Arellano Sota C. (editores.) Tecnologías para la producción de ovejas tropicales. México: FAO; 1989. p. 51-63. 